\title{
Como a Cultura Organizacional Influencia a Evolução de BPM
}

\section{Alternative Title: How Organizational Culture influences BPM Evolution}

\author{
Iveruska Jatobá \\ Centro de Informática(CIn) /UFPE \\ Av. Jornalista Aníbal Fernandes, $\mathrm{s} / \mathrm{n}$ \\ 50.740-560 - Recife - PE \\ icjba@cin.ufpe.br
}

\author{
Carina Alves \\ Centro de Informática(CIn) /UFPE \\ Av. Jornalista Aníbal Fernandes, $s / n$ \\ 50.740-560 - Recife - PE \\ cfa@cin.ufpe.br
}

\begin{abstract}
RESUMO
Gestão de Processos de Negócio (BPM) tem se tornado uma poderosa ferramenta para aumentar a eficiência e eficácia dos serviços prestados nas organizações públicas e privadas. Entretanto, existe uma crescente consciência que BPM requer uma visão holística da organização, onde a cultura organizacional é um fator crítico de sucesso para a implantação de uma iniciativa de BPM. Neste artigo apresentamos um estudo de caso que explora os valores e aspectos culturais de uma organização que contribuem como facilitadores e barreiras para a evolução de uma iniciativa de BPM.
\end{abstract}

\section{Palavras-Chave}

Gestão de Processos de Negócio (BPM), Cultura Organizacional, Cultura de BPM.

\begin{abstract}
Business Process Management (BPM) has become a powerful tool to increase the efficiency and effectiveness of services provided in public and private organizations. However, there is growing awareness that BPM requires a holistic view of the organization where the organizational culture is a critical success factor for the implementation of a BPM initiative. This article presents a case study that explores the values and cultural aspects of an organization that contribute as facilitators and barriers to the development of a BPM initiative.
\end{abstract}

\section{Categories and Subject Descriptors}

K6 [Gerenciamento de Computação e Sistemas de Informação]: Diversos.

\section{General Terms}

Management.

\section{Keywords}

Business Process Management (BPM), Organizational Culture, BPM Culture.

Permission to make digital or hard copies of all or part of this work for personal or classroom use is granted without fee provided that copies are not made or distributed for profit or commercial advantage and that copies bear this notice and the full citation on the first page. To copy otherwise, or republish, to post on servers or to redistribute to lists, requires prior specific permission and/or a fee.

SBSI 2015, May 26-29, 2015, Goiânia, Goiás, Brazil.

Copyright SBC 2015

\section{INTRODUÇÃO}

Todas as organizações desejam otimizar seus serviços, eliminar perdas, melhorar qualidade, aumentar produtividade, reduzir custos, evitar retrabalho e prospectar oportunidades. Nos últimos anos, muitas organizações públicas e privadas têm investido de forma significativa em iniciativas de gestão de processos de negócio (do inglês: Business Process Management - BPM) para alcançar estes objetivos. Muitos projetos de BPM têm originado mudanças significativas nas organizações, que incluem aumento da eficiência operacional, novas ofertas de serviços, automação de processos, redesenho da estrutura organizacional e conformidade regulatória, dentre outros [23].

Entretanto, a consolidação da gestão de processos de negócio é dificultada pelo fato desta disciplina ainda carecer de uma abordagem de gerenciamento unificada e ampla. Dentre as principais dificuldades reportadas por estudos na área podemos destacar a necessidade de mudanças constantes que as organizações precisam acompanhar [13]. De fato, a essência de BPM envolve ciclos de melhorias contínuas, onde a organização e as pessoas precisam adaptar-se às novas formas de trabalho. Em geral, pessoas são resistentes à mudança, onde esta resistência pode ser um dos principais entraves para iniciativas de BPM.

A disciplina de BPM ainda é frequentemente associada às novas tecnologias voltadas para a modelagem e automação de processos de negócios. Entretanto, pesquisas recentes sugerem a adoção de uma abordagem mais holística para as iniciativas de BPM. Esta nova visão inclui aspectos organizacionais e percebe processos como o núcleo central a partir do qual os negócios são realizados por pessoas dentro da organização [9]. Assim, BPM é cada vez mais considerada como uma abordagem de gestão integrada que inclui dimensões como alinhamento estratégico, governança, pessoas e cultura, além de tecnologia de informação (TI), ferramentas e técnicas [13]. Em particular, cultura é considerada como um dos principais impulsionadores de iniciativas de BPM, representando uma fonte importante para o seu fracasso ou sucesso. Neste contexto, a cultura organizacional é cada vez mais reconhecida como um fator central influenciando e sendo influenciada por BPM [24].

Apesar do crescente reconhecimento da importância da cultura como fator crítico de sucesso em iniciativas de BPM, ainda existem poucas pesquisas relacionadas ao tema. Alguns autores [24], [3] resumem as seguintes percepções de cultura encontradas em pesquisas na área de BPM: 
- Influência da cultura organizacional em BPM;

- Influência de BPM na cultura organizacional;

- Cultura de BPM, que descreve uma cultura que suporta os objetivos de BPM;

A partir das motivações descritas acima, este artigo tem como objetivo investigar como a cultura organizacional influencia iniciativas de BPM. Para isso, apresentamos a seguinte questão de pesquisa:

QP1: Quais valores ou aspectos culturais de uma organização contribuem como facilitadores e barreiras para a evolução de uma iniciativa de BPM?

QP2: Qual o impacto de BPM na cultura organizacional?

A fim de responder às questões de pesquisa, a principal contribuição deste artigo é apresentar um estudo de caso exploratório que investiga como os valores ou aspectos culturais de uma organização pública influenciam a sua iniciativa de BPM.

Este artigo é estruturado da seguinte forma. A Seção 2 apresenta a revisão de literatura relacionada ao tema. Na Seção 3 descrevemos o método de pesquisa utilizado. A Seção 4 apresenta os resultados do estudo de caso. Na Seção 5 apresentamos uma discussão sobre os resultados obtidos e descrevemos as limitações da pesquisa. Finalmente, a Seção 6 conclui o artigo e apresenta diretrizes para trabalhos futuros.

\section{REVISÃO DA LITERATURA}

\subsection{BPM}

De acordo com o CBOK [1], a Gestão de Processos de Negócio (BPM) pode ser vista como uma disciplina gerencial que integra estratégias e objetivos de uma organização com expectativas e necessidades de clientes, por meio do foco em processos ponta a ponta. BPM engloba estratégias, objetivos, cultura, estruturas organizacionais, papéis, políticas, métodos e tecnologias para analisar, desenhar, implementar, gerenciar desempenho, transformar e estabelecer a governança de processos. Para Jeston e Nellis[9], BPM pode ser definido como a realização dos objetivos de uma organização através da melhoria, gestão e controle dos seus processos de negócios considerados essenciais.

O entendimento do conceito de BPM difere largamente na academia. Varia desde uma visão técnica, considerada mais estreita, até uma visão mais ampla, com foco em uma perspectiva holística [3]. Esta visão mais abrangente considera que a perspectiva organizacional envolvendo cultura e aspectos humanos são fundamentais para o sucesso de BPM [4],[5].

\subsection{Cultura}

Cultura é um conceito bastante amplo, uma vez que é associado de formas diferentes dependendo do seu contexto [24]. Para Schein [19], cultura é um conjunto básico de premissas tácitas sobre como o mundo é e deve ser, que um grupo de pessoas compartilham e que determina suas percepções, pensamentos, sentimentos, e, até certo ponto, o seu comportamento. É compreendida como o conjunto de valores compartilhados de um grupo que tornam-se visíveis em ações e estruturas [18]. Já Hofstede [6] considera cultura como uma programação coletiva da mente. Valores culturais são centrais para o entendimento de uma cultura. Enquanto comportamentos podem ser modificados através de incentivos, valores são aspectos relativamente mais estáveis ao longo do tempo [8]. De maneira geral, cultura referese aos valores compartilhados por um grupo de pessoas, como nações, organizações e grupos de trabalho, que se tornam visíveis nas ações individuais e coletivas dos membros do grupo [24].

Schein [17] define cultura organizacional como um padrão de premissas básicas inventadas, descobertas ou desenvolvidas por um dado grupo, enquanto este grupo aprende a lidar com os problemas externos e de integração interna. Se essas premissas se provarem válidas, serão ensinadas para os novos membros como a forma correta de perceber, pensar e sentir em relação aos problemas, como parte de um processo de socialização. Com base nesse conceito, uma cultura organizacional pode ser analisada em três diferentes níveis - artefatos, valores e normas, e premissas básicas - de acordo com seu grau particular de consciência e visibilidade, conforme ilustrado na Figura 1.

Artefatos são estruturas visíveis e processos dentro da organização. São diretamente observáveis, mas difíceis de decifrar e incluem tecnologias, símbolos, arquiteturas, linguagens dentro da organização. O segundo nível, representado pelas normas e valores compartilhados, representam as estratégias e filosofias da organização, que transmitem um sentimento do que é certo e errado. Normas e valores servem como um framework de orientação que influencia as ações dos membros dentro da organização. O terceiro nível, consiste em concepções e premissas básicas inconscientes. São vistas como a referência inicial sobre os quais os valores e ações se baseiam [3].

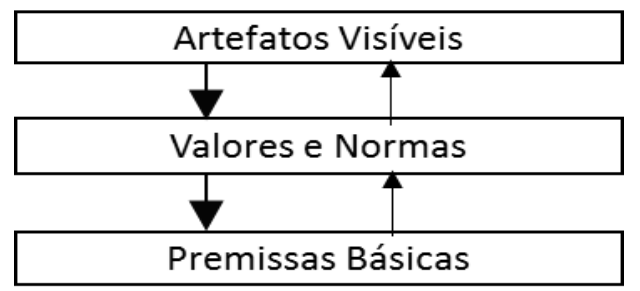

Figura 1. Modelo de Cultura Organizacional de Schein.

\subsection{Cultura de BPM}

Considerando que cultura é um fator crítico de sucesso em iniciativas de BPM e que é predominantemente definida através de valores básicos que estão implícitos em artefatos visíveis, a compreensão de quais valores culturais são considerados impulsionadores de BPM é essencial [15], [24].

Diante desse contexto, vom Brocke \& Sinnl [24] definiram um Modelo conceitual de Cultura de BPM, conforme ilustrado na Figura 2. Neste modelo, Cultura de BPM é compreendida como uma cultura que suporta o desenvolvimento de processos de negócio eficientes e eficazes. Enquanto Contexto Cultural referese à cultura organizacional existente no início de uma iniciativa de BPM. Já Alinhamento Cultural representa a congruência necessária entre a Cultura de BPM e o Contexto Cultural para que uma iniciativa de BPM seja bem sucedida.

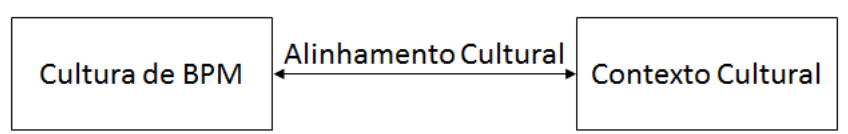

Figura 2. Modelo de Cultura de BPM.

Os autores identificaram um conjunto de valores culturais que suportam os objetivos para estimular uma Cultura de BPM. Esses valores culturais, denominados valores $\boldsymbol{C E R T}$, estão associados a 
fatores organizacionais que são expressos em: Customer Orientation, representados por características de proatividade e capacidade de resposta; Excellence, através de iniciativas de melhoria continua e inovação; Responsibility, por compromisso e responsabilização nos resultados; e Teamwork, através da colaboração horizontal, não departamentalizada. Desta forma, a proposta de Schimiedel [21] é identificar o quanto esses valores estão amadurecidos nas organizações e elaborar estratégias para desenvolver uma cultura organizacional a fim de suportar a implantação de iniciativas de BPM, aumentando as taxas de sucesso. Nesta pesquisa, utilizamos esta proposta como referencial teórico para apoiar o planejamento e execução do estudo de caso. Na seção a seguir apresentamos o método de pesquisa adotado.

\section{MÉTODO DE PESQUISA}

O objetivo desta pesquisa é analisar como a cultura organizacional de uma organização afeta os resultados de uma iniciativa de BPM. Em particular, pretendemos investigar como valores culturais observados na organização estudada atuarão como facilitadores e barreiras para a evolução da iniciativa de BPM. Considerando as questões de pesquisa apresentadas na Seção 1, adotamos um estudo de caso exploratório como estratégia de pesquisa. A unidade de análise foi a iniciativa de BPM conduzida na organização. O quadro metodológico utilizado está apresentado na Tabela 1.

Tabela 1. Quadro Metodológico da Pesquisa

\begin{tabular}{|c|c|}
\hline Concepção Filosófica & Construtivista/Interpretativa \\
\hline Método Científico & Indutivo \\
\hline Natureza & Qualitativa \\
\hline Caráter & Empírico \\
\hline Estratégia de Pesquisa & Estudo de Caso \\
\hline Quanto ao Propósito & Exploratório \\
\hline $\begin{array}{l}\text { Procedimentos de Coleta } \\
\text { de Dados }\end{array}$ & $\begin{array}{l}\text { 1. Entrevistas Semiestruturadas; } \\
\text { 2. Análise de Documentos; } \\
\text { 3. Observação Participante; }\end{array}$ \\
\hline $\begin{array}{l}\text { Procedimentos de } \\
\text { Análise }\end{array}$ & $\begin{array}{l}\text { Classificação temática e análise } \\
\text { qualitativa de entrevistas, } \\
\text { documentos e registros de } \\
\text { observação participante. }\end{array}$ \\
\hline Estratégia de Validação & Triangulação de Métodos \\
\hline
\end{tabular}

Como procedimentos de Coleta dos Dados, utilizamos entrevistas semiestruturadas, análise de documentos e observação participante. Foram realizadas entrevistas com colaboradores da organização diretamente envolvidos na iniciativa de BPM. Para a análise de documentos, obtivemos acesso a toda a documentação produzida pela iniciativa de BPM nos últimos 3 anos. Já a observação participante foi conduzida pelas autoras, já que elas estão envolvidas como consultoras externas da iniciativa de BPM. Conduzimos a observação durante reuniões e atividades de trabalho semanais durante o período de 04 meses. Fizemos uso de um diário de pesquisa para registrar eventos e conversas que consideramos relevantes para condução do estudo de caso.

$\mathrm{Na}$ etapa de Análise dos dados, utilizamos procedimentos de análise temática, onde os dados foram transcritos e codificados utilizando as ferramentas Express Scribe Transcription e Weft QDA, respectivamente. Foram identificadas categorias que sintetizam os principais fenômenos identificados durante o estudo. Os fenômenos analisados referem-se aos valores culturais identificados na organização que influenciam a iniciativa de BPM.

Durante a análise dos dados foi realizada uma classificação das categorias, considerando como critério na definição das categorias o grau de influência que os fatores observados na cultura organizacional exercem na iniciativa de BPM. A partir disso, elaboramos uma lista das categorias consideradas como fatores da cultura organizacional com maior impacto na iniciativa de BPM.

A lista de categorias foi priorizada de acordo com a sua relevância, que foi verificada através da sua ocorrência em várias entrevistas e também reportada no diário de observação. Pudemos identificar estes aspectos da cultura organizacional que impactam a sua iniciativa de BPM: comunicação, inovação, mudança, política, motivação, gestão de pessoas, melhoria contínua, orientação ao cliente, colaboração, gestão organizacional e, num processo cíclico da influência mútua, a própria iniciativa de BPM como indutor de mudança da cultura organizacional.

\section{RESULTADOS}

Esta seção descreve o contexto da organização onde foi realizado o estudo de caso, bem como o perfil dos entrevistados. Além disso, apresenta os principais resultados obtidos para responder às questões de pesquisa. Os resultados foram divididos de acordo com as categorias descritas na seção anterior.

\subsection{Contexto da Organização e da Iniciativa de BPM}

A organização analisada é uma instituição pública que tem como principal objetivo apoiar o controle da gestão pública em benefício da sociedade, atuando como instrumento efetivo na defesa do interesse social e no combate à corrupção.

A iniciativa corporativa de BPM na organização teve início em 2012. Antes disso existiram esforços de modelagem de processos que ocorreram de forma isolada em alguns setores da organização. A adoção de uma filosofia de BPM nesta instituição foi principalmente guiada pelos seguintes objetivos: (i) criação e estruturação de um Escritório de Processos (Business Process Management Office) e (ii) implementação de fluxos de trabalho eletrônico de processos finalísticos da instituição. Dentre os principais resultados alcançados pela iniciativa de BPM, podemos destacar a definição de uma metodologia de BPM alinhada com os objetivos e características da organização, formalização do Escritório de Processos como unidade organizacional, aquisição de uma ferramenta de apoio aos processos finalísticos da instituição. Podemos considerar que a iniciativa de BPM tem evoluído de forma satisfatória, uma vez que ela está atingindo os objetivos definidos pelos patrocinadores. No entanto, identificamos diversos desafios que a organização precisa superar para garantir a evolução desta iniciativa.

Durante o estudo de caso, realizamos entrevistas semiestruturadas com 10 colaboradores da instituição, com os seguintes perfis:

Analista de Sistemas - Trabalha há 5 anos na instituição na área de TI. Tem curso superior em Ciência da Computação.

Analista de Processos - Trabalha há quase 10 anos na instituição, tendo ingressado originalmente na área de TI, mas passando por diversas áreas finalísticas da organização, assim como nas áreas 
de gerenciamento de qualidade, gerenciamento de projetos, atendimento e suporte de TI, dentre outras. Possui Mestrado em Engenharia de Software, além de certificações CBPP (Certified Business Process Professional) e PMP (Project Management Professional).

Analista de Negócio - Trabalha há quase 10 anos na instituição, com formação superior em Engenharia Elétrica. Tem grande conhecimento dos processos finalísticos da organização, atuando neste momento como suporte às áreas de negócio, definindo procedimentos e metodologias de trabalho.

Assessor da Presidência - Trabalha há mais de 23 anos na instituição, atuando em diferentes áreas finalísticas e de suporte, ocupando tanto funções operacionais como funções de gerência e diretoria. Possui especialização na área de Auditoria.

Assessor Técnico - Trabalha há 20 anos na instituição, em diferentes áreas consideradas finalísticas e de suporte/apoio, ocupando tanto funções operacionais como funções de gerência e diretoria na instituição assim como em outros órgãos de governo. Apresenta Mestrado na área de Gestão Pública.

Gerente Funcional - Trabalha há 18 anos na instituição, atuando em diferentes funções operacionais e gerenciais em áreas finalísticas e de suporte. Tem mestrado na área de Direito, sua formação original, e possui experiência na área de BPM.

Gerente de Projeto de BPM - Trabalha há mais de 19 anos na instituição, atuando nos últimos anos em funções gerenciais e diretoria em diferentes áreas da organização. Atualmente é gerente de um dos principais projetos de BPM em condução na organização.

Diretor de Planejamento - Trabalha há 5 anos na instituição, sendo originalmente da área de TI, exerceu funções de gerente de projeto e diretoria na área de suporte da organização. Tem mestrado em Ciência da Computação, na área de gerenciamento de projetos. Possui a certificação PMP (Project Management Professional) em gerenciamento de projetos.

Diretor de TI - Trabalha há 7 anos na instituição, sempre atuando na área de TI. Exerceu funções operacionais de analista de sistemas, bem como gerenciais.

A seguir descrevemos as principais categorias identificadas no estudo de caso, que apresentam diversas características culturais afetam a sua iniciativa de BPM.

\subsection{Comunicação}

A categoria Comunicação diz respeito à transmissão de informação relevante, ou seja, o compartilhamento da informação, dentro e para fora da organização. Observamos na organização que a comunicação, tanto interna (entre setores) quanto externa (com outros órgãos e sociedade) diretamente relacionada à atividade finalística, é identificada como um dos pontos fracos da organização. Essa constatação está diretamente associada à existência de canais de comunicação falhos e à necessidade de modernizar e profissionalizar a comunicação da instituição.

Além disso, outros fatores também contribuem para essa fragilidade na comunicação externa, como a própria área de atuação da instituição, que é de difícil compreensão pela população em geral.

Observamos também a inexistência de uma estratégia de comunicação interna, tendo como consequência a dificuldade em compartilhar a informação entre os setores da organização, seja pela falta de agilidade, pela fragilidade dos canais existentes ou pelo próprio desconhecimento do fato gerador da informação pela área de comunicação.

Diante desse contexto, foi constatada uma grande preocupação com a melhoria da comunicação, através de ações como a profissionalização do setor responsável e criação de um objetivo estratégico relacionado ao tema. Com isso, já se evidencia a percepção de uma melhoria significativa da comunicação externa, com rebatimento direto na comunicação interna. O Diretor de Planejamento destaca que "Eu tenho visto uma melhora significativa nos últimos anos. Mais do que uma melhora, uma preocupação em se comunicar melhor... A organização tem se preocupado com isso, buscado uma profissionalização principalmente na comunicação externa, mas que tem rebatimento na comunicação interna... Tanto que temos um objetivo estratégico de fortalecimento da imagem institucional".

\subsection{Mudança}

A categoria Mudança refere-se à forma como a organização reage às mudanças, se há incentivo à quebra de paradigmas, bem como flexibilidade na adaptação da sua cultura organizacional. Observamos como valor cultural uma forte resistência à mudança, representado por um sentimento de desconfiança dos funcionários ao novo, especialmente quando são tirados da sua zona de conforto. De acordo com o Analista de Sistemas: "Para tudo que estamos implantando aqui existe uma resistência: por que mudar? Isso vai me dar mais trabalho... Além disso, vou ter que fazer mais isso".

Outro aspecto gerador da resistência às mudanças dentro da organização é o choque de gerações. Como se existissem duas instituições, conflitantes, em uma única instituição formal. Os funcionários mais recentes entraram, em sua maioria, com desejo de colaborar para o desenvolvimento de uma gestão mais moderna. Já os mais antigos, que talvez um dia tenham tido os mesmos anseios, mostram-se mais reativos, sempre procurando defeitos nas propostas de mudança. Segundo o gerente funcional "Para fazer uma mudança aqui é complicado. Entraram muito jovens, com 18 ou 20 anos. As pessoas são contaminadas. Você entra aqui com vontade de fazer acontecer, então antigamente as pessoas tinham iniciativas individuais, e nos primeiros obstáculos se decepcionavam e se acomodavam...".

Entretanto, apesar da resistência às mudanças ser uma característica intrínseca à cultura organizacional da instituição analisada, esta resistência pode ser minimizada pelo poder político que incentiva e apoia a mudança. Ou seja, existe a necessidade de patrocínio da alta gestão com o objetivo de influenciar e facilitar as ações de mudança, conforme descrito pelo Diretor de TI “... Se não houver o patrocínio do presidente ou da gestão para uma mudança substancial, ela não ocorre".

Outro elemento facilitador identificado é o envolvimento de pessoas para quebrar a resistência. Trabalhar a efetividade da comunicação, como as pessoas irão perceber e compreender a mudança proposta, e, principalmente, como elas serão beneficiadas, é fundamental para conseguir o apoio e quebrar os silos de resistência, garantindo um ambiente favorável à transformação. Conquistar os funcionários da instituição é o maior desafio de um processo de mudança organizacional

No caso específico da iniciativa de BPM, por ser um agente de transformação organizacional, com o objetivo de neutralizar a barreira identificada, sugere-se o envolvimento das pessoas nas discussões sobre melhoria de processos, mesmo que implique 
num trabalho maior de gerenciamento das pessoas envolvidas. Esse fato pode ser verificado nos trechos das entrevistas com o Diretor de Planejamento e Assessor da Presidência, respectivamente: "O excesso de formalismo e a resistência à mudança podem impactar a iniciativa de BPM..." $e$ "...Se você envolve as pessoas na discussão, ainda que você tenha um público maior na implantação do processo, em compensação facilita muito o processo de implantação...".

\subsection{Gestão de Pessoas}

Embora o significado do termo Gestão de Pessoas seja muito mais amplo, no contexto desta pesquisa, esta categoria refere-se ao modo como a organização identifica e estimula o desenvolvimento das competências e habilidades dos seus funcionários. Como a organização percebe e trata seus sentimentos, emoções, comportamentos no ambiente profissional, bem como adota políticas de valorização e estímulo à meritocracia.

Observamos que não existe uma política formal estabelecida pela área de gestão de pessoas para tratar de forma institucional o desenvolvimento das competências e habilidades dos funcionários. Embora gestões anteriores tenham criado um banco de talentos para identificar e armazenar as competências e habilidades de seus funcionários, constatou-se que essa ferramenta não teve seu uso amplamente difundido dentro da organização. Dessa forma, a alocação das pessoas é realizada informalmente com base no conhecimento pessoal ou na indicação direta ou indireta aos gerentes das áreas. Com isso, percebe-se desperdício no aproveitamento das competências e habilidades existentes na organização. Essa questão pode ser ressaltada pelo trecho de entrevista com o Diretor de Planejamento: "Quando algum gestor tem conhecimento de alguma habilidade de algum servidor tenta, na maioria das vezes, fazer o bom uso. Mas por vezes a gente desperdiça uma habilidade ou competência por desconhecer...".

Em relação ao relacionamento interpessoal, constatamos a existência de conflitos internos provocados por disputas salariais e de poder entre funcionários de diferentes categorias. Isso tem gerado ao longo dos anos um desgaste grande entre os funcionários e acaba prejudicando a harmonia no ambiente de trabalho. A alta gestão, apesar de ciente, não intervém. No entanto, como essa questão acaba interferindo na produtividade, tenta-se contornar esse problema através de conscientização sobre a ética no trabalho e da meritocracia, premiando quem atinge as metas e os resultados. Isso pode ser confirmado no trecho da entrevista com o Gerente Funcional: "E a organização hoje vive em conflito entre os diferentes cargos, todos fazem a mesma coisa $e$ pleiteiam salários iguais. A organização tende a não se contrapor a ninguém, a não se posicionar, a deixar que os servidores se resolvam. É uma briga que termina refletindo na produtividade... Então, o clima hoje é tenso, já foi muito melhor".

Quanto à motivação dos funcionários, percebemos o orgulho de trabalhar na organização pela sua missão, considerada nobre, podendo contribuir diretamente na maior eficiência da gestão pública. Além disso, os funcionários também sentem orgulho da excelente capacidade técnica do quadro funcional da instituição, da autonomia na execução do trabalho e da remuneração salarial. Segundo o Analista de Negócio: "Há uma satisfação das pessoas pelo salário, pela estrutura e pela função da organização". Todavia, apesar dos fatores motivacionais relatados, foi reportada uma acomodação por parte de alguns servidores, seja por desgaste e cansaço da vida profissional ou por não conseguir enxergar um resultado efetivo do seu trabalho, por exemplo. Esse fato é agravado pelo fato dos gerentes não contarem com o apoio de ferramentas institucionais para gerir suas equipes de forma padronizada e efetiva. Essa dificuldade gerencial é confirmada pelo Assessor Técnico: "Os gerentes sentem muita falta do apoio da diretoria de gestão de pessoas... O gerente fica muito à mercê, e se queima muito. Porque nem tudo está normatizado em termos de gestão de pessoas... Termina aquele gerente que é mais rígido, ou porque que ele quer que a coisa vá mais rápido, sendo um gerente chato. Então não é fácil você ser um gerente que cobra metas, que tem planejamento, que quer agilidade". Devido às relações hierárquicas serem dinâmicas, especialmente em órgãos públicos, é também muito comum o receio dos gerentes na forma de se relacionar com seus subordinados, pelo fato de que hoje são gerentes, mas amanhã podem se tornar subordinados, numa inversão de papéis.

Ainda em relação à acomodação dos funcionários, o concurso público e a consequente estabilidade são considerados como um motivador da fragilidade do comprometimento com as metas $e$ resultados organizacionais. Com isso, percebe-se a necessidade de implantação de uma política de recompensa e promoção com base na avaliação de desempenho dos funcionários. Segundo o Analista de Negócio: "É como na empresa privada, tem uma seleção, mas você tem que mostrar resultado... Basicamente é dar consequências mais fortes em relação à promoção. Deixar a pessoa que não está trabalhando desconfortável".

A política de recompensa é uma das ações da organização relacionadas à meritocracia. No entanto, ainda não existe a possibilidade de uma remuneração variável com base na produção e não mais pela antiguidade. Além disso, com a gestão cada vez mais orientada a resultados, torna-se imprescindível a ocupação das funções estratégicas dentro da instituição por pessoas com comprovada competência e comprometimento, conforme ressaltado pelo Analista de Processos: “... À medida que [a organização] está reforçando essa questão de objetivos, de metas, então, cada vez mais as pessoas estão buscando colocar pessoas competentes em cargos importantes, restando aos protegidos aquelas funções em que ele vai ganhar uma gratificação mas que o papel dele não vai ser tão importante... Como as pessoas em cargos de chefia terminam tendo uma influência grande no atingimento ou não dessas metas, então está cedendo espaço cada vez mais para a meritocracia". Dessa forma, podemos perceber uma valorização à meritocracia.

\subsection{Colaboração}

A categoria Colaboração explora o modo como as diferentes áreas da organização cooperam entre si para realizar atividades interdepartamentais, e se apresentam um senso comum de pertencer a um time trabalhando para atingir um objetivo comum.

Observamos que os funcionários, de uma forma geral, não apresentam uma visão holística dos serviços prestados pela organização e como cada departamento ou cada uma delas contribui. As pessoas não se sentem parte efetiva de um time, demonstrando claramente a baixa integração entre as equipes. Os departamentos trabalham como ilhas isoladas na execução de suas atividades. Estão preocupados apenas com os seus resultados individuais, e não com o resultado final da organização. Com isso, a passagem de bastão entre os diferentes setores ocorre de forma precária dificultando a prestação dos serviços finalísticos. Este aspecto foi relatado pelo gerente de projeto de BPM: "Não sei se 
esses valores de equipe, de grupo para os objetivos macros estão internalizados pelas pessoas que estão na área fim. Tanto que às vezes não existe uma compreensão das mudanças que a alta gestão indica... Talvez as pessoas não se sintam parte efetiva. $E$ isso pode impactar negativamente na mudança que está por vir".

Em contrapartida, também percebemos que existe na instituição o incentivo à cultura de projetos e processos como instrumento de colaboração e integração entre equipes. Ações relacionadas à gestão de projetos, gestão por processos, planejamento estratégico e portfólio de projetos e serviços têm melhorado a integração e a criação de equipes multidisciplinares trabalhando como um time, em prol de objetivos comuns que ajudam a atingir as metas estratégicas estabelecidas pela instituição. É fundamental criar uma cultura de estabelecer parcerias entre as diversas áreas, dividindo os riscos, e com a definição clara de responsabilidades entre os diversos atores. Segundo o analista de negócio: "Esse enfoque nos processos é muito importante. As pessoas têm que entender que não é na minha caixinha tal, eu sou um pedaço do processo tal. Isso, de fato, tem que melhorar".

\subsection{Melhoria Contínua}

A categoria Melhoria Contínua refere-se ao modo como a organização incentiva os funcionários à prática de revisar constantemente os processos e procedimentos de trabalho a fim de identificar deficiências e propor melhorias nas suas atividades.

Verificamos uma falta de incentivo à melhoria contínua na organização, uma vez que não faz, parte da sua cultura aprender com os erros, conforme ressaltado pelo Analista de Processos: "Em nenhum momento, não que eu tenha conhecimento, há uma prática de dizer e identificar o motivo pelo qual o projeto deu errado. Talvez ajudasse melhorar tanto a maneira como a casa trabalha conduzindo essas ideias, e aprender porque aquilo não deu certo. Quando as coisas dão erradas a gente tem muita chance de aprender alguma coisa".

Com a elaboração do planejamento estratégico na organização, definição de metas e indicadores, observamos uma preocupação excessiva da instituição com o cumprimento dos prazos em detrimento da qualidade do serviço prestado. Isso se reflete em um tradeoff entre prazo e qualidade, uma vez que as cobranças pelos resultados de produtividade individual medem o quanto os funcionários conseguem executar suas atividades nos prazos estabelecidos, deixando em segundo plano a preocupação com a eficiência do resultado. "A preocupação é com o número. Primeiro é o número, eu sou medido pelo tempo, não sou medido pela qualidade...".

\subsection{Gestão Organizacional}

A categoria Gestão Organizacional está relacionada à definição dos princípios, normas e objetivos da organização, bem como à estratégia que será adotada para atingi-los. Trata de como a instituição propõe ações, gerencia seus recursos e realiza o monitoramento para alcançar os resultados esperados.

Observamos que nem todos os valores descritos no planejamento estratégico são de fato os valores reais da organização. De acordo com o Gerente do Projeto de BPM: "Não sei se os valores que a gente tem atribuídos no nosso planejamento estratégico estão bem internalizados por todos". Porém, desses valores definidos, ética e transparência são motivos de orgulho e considerados elementos enraizados e priorizados pelos funcionários, conforme trecho a seguir: “... a gente prima muito pela transparência $e$ ética" e "honestidade é um valor muito forte, porque se a gente não representar isso, a gente não é nada...".

A vaidade é um valor cultural implícito observado. Vaidade no sentido de se sentir privilegiado e valorizado pelo seu trabalho, quando um servidor ou grupo de servidores faz um trabalho excepcional que traz muitos benefícios para a organização. Mas também pelo aspecto negativo, quando não se apoia uma determinada iniciativa ou ideia porque esta pertence a outro. Como consequência, realizar qualquer transformação ou mudança organizacional exige uma estratégia bem planejada a fim de tratar a questão da vaidade junto às áreas e pessoas impactadas. Conforme Gerente Funcional: "Porque se você aqui brilhar como salvador da pátria, você vai ser estraçalhado. É uma fogueira de vaidades. É melhor que você fique no background...".

Aspectos da cultura organizacional existente, que inclui características como burocracia, legalismo e resistência à mudança são identificadas como barreiras à implantação de uma gestão mais moderna. Com isso, observamos um modelo de gestão obsoleto, onde pessoas tem uma postura conservadora convivendo com uma tentativa de implantar um novo modelo de gestão orientado a resultados.

Assim, a institucionalização da área de planejamento tem sido o grande indutor de desenvolvimento e modernização da organização, através da implantação de um planejamento estratégico, com objetivos e metas bem definidas, norteando as ações de melhoria. Essa dinâmica já está incorporada pela alta gestão. Também verificamos um alinhamento entre os objetivos estratégicos definidos pela instituição e o que a sociedade, seu principal cliente, espera.

A sedimentação dessa cultura de planejamento também é considerada um grande facilitador à implantação de uma gestão por processos na organização, ressaltado pelo Gerente Funcional: "A consolidação da cultura do planejamento, já sedimentada. Se não tivesse isso, haveria um problema sério, grave, porque isso precede qualquer iniciativa de BPM. Sem isso,... eu não tenho para onde ir, não tenho uma diretriz, não tenho uma meta...".

\subsection{BPM como Indutor da Mudança da Cultura Organizacional}

A categoria BPM como indutor da Mudança da Cultura Organizacional refere-se ao modo como a iniciativa de gestão de processos de negócio na instituição pode colaborar com a mudança da cultura organizacional, num processo cíclico de retroalimentação. Ou seja, a cultura organizacional, como um dos fatores críticos de sucesso da implantação de BPM, sofre transformações ao longo do seu ciclo de implantação, transformações estas que podem facilitar a consolidação de BPM.

Uma das barreiras identificadas é a necessidade da alta gestão $\boldsymbol{e}$ funcionários entenderem como BPM contribui para melhoria da organização. Em geral, observamos que as pessoas podem não saber o significado de BPM, ou sua primeira percepção é que BPM trata-se apenas de mais uma ferramenta limitada a atividades operacionais, com baixo valor agregado à organização. Sem o devido alinhamento com a estratégia organizacional, e cuja preocupação e responsabilidade deve ser restrita apenas a um conjunto de profissionais que trabalham no escritório de processos. Porém, a gestão por processos trata de algo muito mais amplo e revolucionário que tem o poder de apoiar a transformação da organização. É um instrumento para repensar o negócio e 
apoiar a tomada de decisões. Isso é ressaltado pelo Gerente Funcional: "O que mais me desestimula é as pessoas não entenderem muito bem o que é isso [BPM]: o poder revolucionário dessa proposta. As pessoas não entendem, olham para a gestão por processos hoje como se fosse um trabalho de diagnóstico e não um trabalho de mudança organizacional...".

A demora na apresentação de resultados da iniciativa de BPM na organização também é considerada um dos fatores que tem dificultado a consolidação da gestão por processos. Considerando a cultura existente onde é necessário comprovar os resultados de uma iniciativa para a instituição, o tempo necessário para planejar e estruturar a iniciativa de BPM pode comprometer a sua disseminação na organização. $O$ atraso na apresentação dos resultados ou a sua dissociação com a iniciativa de BPM pode comprometer esse incentivo, representando um grande risco para o projeto. Segundo o Gerente Funcional "Eu sempre percebi que para você conseguir apoio, patrocínio, recurso, você tem que mostrar resultado. Se o resultado não é atribuído a você... Você pode até estar produzindo, mas se o resultado não está sendo reconhecido, não vai voltar em termos de recursos, de apoio...".

Em relação aos fatores que facilitam a transformação da cultura organizacional, observamos que BPM ajuda a organização a se conhecer e discutir seus problemas. O olhar com uma visão crítica sobre a forma de trabalho, questionar o modo como as coisas são feitas e pensar no que pode ser melhorado já é um caminho para a instituição evoluir. Isso implica num maior conhecimento da organização, mesmo quando as discussões tratam de questões polêmicas. “... o fato de estar discutindo o processo com as áreas, facilita para que a área entenda o que ela faz. Antes isso não existia, você trabalhava meio mecânico, executando tarefa, sem nenhuma consciência do que você estava fazendo e qual o seu papel dentro da organização. As pessoas estão parando para discutir os problemas...".

Outro aspecto observado é que a consolidação da gestão por processos é um instrumento para instituir uma visão não departamentalizada, ou seja, uma visão horizontal dos processos. Isto influencia diretamente a importância da colaboração entre as diversas áreas que atuam num mesmo processo, conforme ressaltado pelo Diretor de Planejamento: "O BPM pode nos ajudar de forma significativa a sermos cada vez mais ágeis na prestação de serviços, e mais, ele também permite a gente quebrar as barreiras no que se refere à colaboração interna entre as áreas, e ter essa visão do todo. A visão de que a organização existe para receber um insumo e gerar valor para a sociedade".

\section{DISCUSSÃO}

Considerando a própria iniciativa da gestão de processos de negócio na instituição como indutora de mudança da cultura organizacional, observamos que vários aspectos culturais identificados como barreiras e facilitadores, sofreram um processo de evolução, à medida que o ciclo de implantação de BPM se desenvolveu. Isso demonstrou uma influência cíclica e contínua entre a cultura organizacional e a iniciativa de BPM, facilitando a consolidação da gestão de processos de negócio na organização. Também percebemos que várias características culturais identificadas durante a pesquisa, se relacionam, contribuindo para mitigar os efeitos negativos ou desenvolvendo os positivos.

Em relação à necessidade da alta gestão e funcionários entenderem como BPM contribui para a melhoria da organização, observamos que os aspectos culturais relacionados à comunicação, mudança e à gestão de pessoas, especialmente os $\mathrm{s}$ relacionados ao desenvolvimento das competências e habilidades, são fundamentais e precisam ser desenvolvidos para colaborar com a implantação do BPM.

A limitação dos canais de comunicação dificulta a divulgação, com clareza e tempestividade, de informações e resultados das ações da organização. Neste caso, é considerada uma barreira à compreensão pela alta gestão e funcionários sobre o real propósito da implantação do BPM. Ciente desse cenário, percebemos que a organização apresenta preocupação e definiu ações, relacionadas à melhoria da comunicação, para modificar esse contexto.

A melhoria da comunicação interna também contribui para que o projeto de BPM seja utilizado como instrumento para instituir uma visão mais colaborativa na execução dos seus processos primários. A consequência dessa mudança na visão das responsabilidades e do papel de cada setor e indivíduo na execução dos processos pode resultar num maior comprometimento para o atingimento das metas institucionais, consolidando uma gestão orientada a planejamento e a resultados. Para isso, a consolidação de uma política de recompensas, assim como o orgulho de trabalhar na instituição, também contribuem positivamente para desenvolver os aspectos culturais relacionados ao comprometimento, motivação e colaboração.

Esse cenário fomenta a organização e seus integrantes a desenvolverem uma visão crítica e a discutir seus problemas. E, principalmente, a querer resolvê-los, aprendendo a partir dos erros identificados, quebrando, aos poucos, a burocracia e o legalismo, bem como os silos de poder político e de resistência às mudanças. $\mathrm{O}$ desenvolvimento da característica de aprendizagem organizacional deverá apoiar o crescimento do aspecto cultural relacionado à melhoria contínua.

A demora na apresentação de resultados constitui um grande risco ao projeto de BPM, e pode comprometer o entendimento pela alta gestão e pelos funcionários da importância da iniciativa para a transformação da organização. Isso dificulta a expansão da iniciativa na organização, uma vez que implica numa provável perda de patrocínio político e, consequentemente, de recursos.

Os conflitos internos por disputas salariais e de poder, bem como a resistência às mudanças constituem barreiras que precisam ser neutralizadas, pois influenciam negativamente a iniciativa de BPM. Com isso, observamos um impacto na apresentação de resultados rápidos e tangíveis, e, consequentemente na compreensão pelos funcionários e alta gestão de como o BPM pode contribuir para transformar a organização.

\section{CONCLUSÃO E TRABALHOS FUTUROS}

Este artigo apresentou uma análise sobre quais valores e aspectos culturais de uma organização pública influenciam uma iniciativa de BPM como facilitadores ou barreiras. Assim, nossa pesquisa serve para alertar as diferentes organizações que pretendem iniciar ou que estão em processo de implantação de iniciativas de BPM sobre a importância de identificar quais dos seus valores organizacionais poderão contribuir de forma positiva ou negativa com os projetos de BPM. A partir dessa compreensão, é possível mitigar os possíveis impactos negativos e desenvolver aqueles valores culturais que atuam como facilitadores de uma cultura de BPM. A pesquisa também pode contribuir para organizações que apresentem semelhanças ao contexto cultural analisado, uma vez que alguns dos aspectos culturais apresentados, e que contribuem 
como facilitadores ou barreiras, podem ser encontrados em outras organizações públicas brasileiras.

Com o objetivo de identificar os valores e aspectos culturais que influenciam a iniciativa de BPM na organização em análise, esta pesquisa foi embasada em alguns trabalhos como a pesquisa de Schmiedel e vom Broke, que define a existência de uma Cultura de BPM e a necessidade de alinhamento cultural de uma organização a esse modelo, através do desenvolvimento dos valores CERT [19], [20], [21], [24]. Também foi considerada no nosso trabalho a pesquisa proposta por Mörmann [3], que trata sobre a relação entre BPM e Cultura Organizacional, lançando uma visão crítica sobre a necessidade de abordar também a perspectiva da psicologia organizacional. Com isso, a gestão de pessoas através das relações interpessoais, as emoções e sentimentos do grupo também devem ser levadas em consideração na análise da relação entre cultura organizacional e seu impacto nas iniciativas de BPM.

Uma limitação desse estudo é o foco em um único contexto cultural, com características bem específicas de uma organização pública. Como trabalho futuro, iremos associar os aspectos culturais identificados na organização com os valores CERT, definidos no modelo proposto por Schmiedel e vom Brocke [19], indicando uma contribuição positiva ou negativa ao desenvolvimento de uma cultura de BPM. Também iremos apresentar um conjunto de ações na organização estudada para mitigar os valores culturais identificados como barreiras e estimular aspectos culturais considerados facilitadores.

\section{REFERENCIAS}

[1] CBOK. 2013. Guia para o Gerenciamento de Processos de Negócio: Corpo Comum de Conhecimento. Versão 3.0, $1^{\text {a }}$. Ed. ABPMP. Brasil.

[2] Easterbrook, S., Singer, S., Storey, M. and Damian, D. 2008. Selecting Empirical Methods for Software Engineering Research. In: Guide to Advanced Empirical Software Engineering, eds. Springer London.

[3] Grau, C., Mörmann, J. 2014. Investigating the Relationship between Process Management and Organizational Culture:Literature Review and Research Agenda. Sciedu Press, v.1, n.2.

[4] Hammer, M. 2010. What is business process management. In: vom Brocke, J.; Rosemann, M.: Handbook on Business Process Management 1 - Introduction, Methods, and Information Systems. Springer-Verlag, Berlin Heildelberg.

[5] Harmon, P. 2010. The scope and evolution of business process management. In: vom Brocke, J.; Rosemann, M.: Handbook on Business Process Management 1 Introduction, Methods, and Information Systems. SpringerVerlag, Berlin Heildelberg.

[6] Hofstede, G. 2005. Culture and Organizations: Software of the Mind. McGraw-Hill. New York.

[7] Hribar, B., Mendling, J. 2014. The Correlation of Organizational Culture and Success of BPM Adoption. Twenty Second European Conference on Information Systems, Tel Aviv.

[8] Jayaganesh, M., Shanks, G. 2009. A Cultural Analysis of Business Process Management Governance In Indian Organisations. 17th European Conference on Informationa Systems, ECIS 2009, Verona.
[9] Jeston, J., Nelis, J. 2008. Business Management by Process: Pratical Guidelines to Successful Implementations. Elsevier, Oxford, $2^{\circ}$. Ed.

[10] Jeston, J., Nelis, J. 2008b. Management by Process: A Roadmap to Sustainable Business Process Management. Elsevier, Oxford.

[11] Marconi, M.A., Lakatos, E.M. 2004. Metodologia Científica. Atlas, 4 ed. São Paulo.

[12] Merrian, S.B. 2009. Qualitative Research: a guide to design and implementation. The Jossey-Bass, 2o. Edition. San Francisco, CA.

[13] Rosemann, M., vom Brocke, J.2010.The Six Core Elements of Business Process Management. In: vom Brocke, J.; Rosemann, M.: Handbook on Business Process Management 1 - Introduction, Methods, and Information Systems. Springer-Verlag, Berlin Heildelberg.

[14] Runeson, P., Höst, M. 2008. Guidelines for conducting and reporting case study research in software engineering. Empirical Software Engineering, v. 14, n.2.

[15] Santos, H.,Santana, A. and Alves, C. 2011. Análise de Fatores Críticos de Sucesso da gestão de Processos de Negócio em Organizações Públicas. VII Simpósio Brasileiros de Sistemas de Informação, 262-273

[16] Santos, H. 2012. Fatores Críticos de Sucesso das Iniciativas de BPM no Setor Público. Dissertação de Mestrado. Centro de Informática, Universidade Federal de Pernambuco.Schein, E.1990. Organizational Culture.American Psychological Association, v.45,n,2.

[17] Schein, E. 2010. Organizational Culture and Leadership. Jossey-Bass, 4o. Edition. San Francisco, CA.

[18] Schein, E.1996. Three Cultures of Management:The Key to Organizational learning.. Sloan Management Review.

[19] Schmiedel, T.,vom Brocke, J., Recker, J. 2012. Cultural Fitness for Business Process Management: What is it and what is it worth? The World Financial Review. DOI= http://www.worldfinancialreview.com

[20] Schmiedel, T.,vom Brocke, J., Recker, J. 2012. Is your organizational Culture Fit for BPM?. Buseness Process Trends.DOI= www.bpm-culture.org

[21] Schmiedel, T.,vom Brocke, J., Recker, J. 2012. Which cultural values matter to business process management?Results from a global delphi study. Business Process Management Journal, v.19, n.2. Emerald.

[22] Spanyi, A. 2003, Business Process Management is a Team Sport. Anclote Press, Tampa, FL.

[23] Tumbas, S., Schimiedel, T. 2013. Developing a Culture Supportive of BPM. 11th International Conference on Wirtschaftsinformatik, Leipzig, Alemanha.

[24] Vom Brocke, J., Sinnl, T. 2011. Culture in business process management: a literature review. Business Process Management Journal, v.17, n.2. Emerald. 